\title{
OTHONOMTCS
}

Revista de economía, empresa y sociedad

Dossier «Revolución 4.0: ¿progreso o precarización?»

Coordinador: Josep Lladós

RETOS DE FUTURO... Y DE PRESENTE

\section{¿Nos robarán los robots los puestos de trabajo? Un vistazo al mercado laboral en España}

\section{Josep Lladós}

Profesor agregado de los Estudios de Economía y Empresa de la UOC

RESUMEN Las tecnologías basadas en la inteligencia artificial y en la robótica son uno de los principales retos actuales sobre el futuro del trabajo humano. La llamada Revolución 4.0 cuestiona algunos modelos de negocio, transforma los requerimientos formativos para el sistema productivo e impacta progresivamente en la distribución de la renta.

El mercado laboral español es un buen ejemplo de cómo esta nueva oleada de cambio tecnológico puede impactar en los niveles y sobre todo en la estructura de la ocupación. Se detecta una creciente polarización en la demanda de trabajo y en las oportunidades de trabajo en función de los niveles educativos y de varios efectos de desajuste laboral, atribuibles principalmente a las características de un modelo productivo dominante que es intensivo en tareas de naturaleza rutinaria, pero poco activo en la incorporación de las tecnologías emergentes.

PALABRAS CLAVE Revolución 4.0; mercado laboral; inteligencia artificial; robótica

\section{Will robots take our jobs? A look at the labour market in Spain}

ABSTRACT The technologies based on artificial intelligence and robotics are one of the leading challenges facing us now with regards to the future of human work. The so-called Industry 4.0 is placing various business models in doubt, transforming training requirements for the system of production, and is progressively impacting on the distribution of profit.

The Spanish labour market provides a good example of how this new wave of technological change can have an impact on the levels and especially the structure of employment. A growing polarisation can be seen in labour demand and employment opportunities in accordance with educational levels and the various effects of labour imbalance, which are principally attributable to the characteristics of a dominant production model that is intensive in tasks of a routine nature but not particularly active in the incorporation of emerging technologies. 
KEYWORDS Industry 4.0; labour market; artificial intelligence; robotics

\section{La relación entre cambio tecnológico y ocupación}

¿Nos dejarán sin trabajo los robots? Esta parece ser la principal inquietud social cuando hablamos de las consecuencias de la llamada cuarta revolución industrial. No es poca cosa, porque indudablemente la digitalización tendrá una influencia capital en el futuro del trabajo, también en nuestra casa.

El cambio tecnológico, sin embargo, no es un proceso lineal y determinista fácil de predecir, ya que no acostumbra a desarrollarse en el tiempo siguiendo pautas muy delimitadas ni tampoco se disemina sistemáticamente entre los diferentes sectores económicos. Por el contrario, a menudo avanza a sacudidas y, a veces, lo hace de manera imprevista. La incidencia de la nueva oleada de automatización probablemente también será progresiva y facilitará tiempo para adaptarse, adquirir los conocimientos necesarios y acomodarse satisfactoriamente a los cambios laborales y organizativos que se deriven.

En realidad, la incorporación de nuevos conocimientos a la actividad productiva es uno de los principales factores de progreso económico y social. De hecho, los adelantos tecnológicos y científicos desarrollados en el último siglo no tienen precedente histórico sin que su aplicación generalizada en la actividad económica haya generado una situación de sustitución masiva de trabajo. Por el contrario, la aplicación continuada de nuevas técnicas y conocimientos ha aumentado las posibilidades de producción y de consumo, a la vez que también lo ha hecho con los niveles de ocupación.

Aun así, una de las preocupaciones recurrentes de las sociedades modernas es conocer los efectos del cambio tecnológico en el mercado laboral. Esta inquietud se hace más patente cuando las sociedades afrontan los riesgos derivados de la revolución digital. Por un lado, porque ha acelerado el ritmo de cambio tecnológico con el desarrollo de numerosas innovaciones y aplicaciones que están transformando profundamente los procesos de producción, distribución y consumo. Por otro lado, porque las innovaciones tecnológicas de base digital también están modificando sensiblemente los conocimientos y las habilidades que se requieren en el trabajo.

Si bien ha quedado patente que el impacto del cambio tecnológico sobre la demanda laboral agregada no es negativo, también se ha puesto de manifiesto que sus consecuencias en la composición del empleo son muy importantes. Las tecnologías emergentes no solo sustituyen y crean ocupación, también transforman el trabajo y modifican su composición. Es decir, el cambio tecnológico no es neutral, más bien induce a unos efectos sesgados sobre la demanda de cualificaciones, porque la tecnología se complementa siempre mejor con unas habilidades que con otras. Y, a pesar de que estas habilidades no tienen por qué ser exclusivas de los niveles educativos superiores, las nuevas tecnologías acostumbran a complementarse mejor con el trabajo de más cualificación. Esta mayor complementariedad provoca que aumente la demanda laboral y mejoren los niveles de productividad de este conjunto de trabajadores, probablemente porque están mejor preparados para aprender los nuevos conocimientos y para adaptarse más rápidamente a los cambios organizativos requeridos por el uso de las tecnologías emergentes.

En realidad, la interacción entre innovación tecnológica y trabajo depende de la combinación de cuatro efectos diferentes que tienen naturaleza directa o indirecta. Obviamente, hay efectos relacionados con la sustitución y la creación directa de trabajo. Por un lado, la aparición de nuevas tecnologías sustituye trabajo, con el objetivo de elevar la productividad y ahorrar costes productivos. Este efecto es lo que se conoce como automatización laboral, ya que permite el desarrollo de tareas sin intervención humana. Por otro lado, el cambio tecnológico crea ocupación en aquellas actividades económicas que son el origen de las innovaciones o que están estrechamente relacionadas. La demanda de estos sectores se expandirá, y con ella sus niveles de ocupación.

Nada nos garantiza que el balance resultante de la interacción de estos dos efectos directos sea necesariamente positivo, pero la experiencia nos muestra que el resultado final agregado es favorable por la presen- 
cia de otros efectos inducidos que compensan la destrucción de ocupación. El primer efecto indirecto tiene relación con la complementariedad existente entre el capital tecnológico y el capital humano. Al aumento en la demanda del trabajo que dispone de los conocimientos específicos incorporados a las nuevas tecnologías, y a la reconfiguración de tareas en cada puesto de trabajo que resulta de su utilización, se añade la aparición de ocupaciones emergentes surgidas de la aplicación económica novedosa de los nuevos conocimientos y habilidades adquiridas. Finalmente, el cambio tecnológico implica también el efecto colateral de impulsar la demanda de bienes y de servicios que son ajenos al desarrollo de las innovaciones. En la medida en que el cambio tecnológico mejora la productividad y las rentas, también se impulsa una mayor demanda en otras actividades económicas que favorece el crecimiento del empleo.

Pero en la medida en que estos efectos indirectos son probablemente más intuitivos que evidentes, el grueso de los estudios de impacto potencial ha concentrado sus esfuerzos esencialmente en estimar las consecuencias negativas de la automatización digital, induciendo una visión alarmista y angustiosa, pero también parcial, de las tecnologías 4.0.

Es importante comprender que las tecnologías que emergen de la innovación tienen efectos proporcionales al alcance con el que se utilizan, de manera que las tecnologías de uso más general (como sería el caso de las digitales) tienen efectos potenciales más amplios que aquellas que solo se aplican en la mejora de algunos procesos específicos. También es sabido que las tecnologías digitales no solo sustituyen habilidades manuales, también reemplazan algunas habilidades mentales. Así, todo haría pensar que la nueva oleada de revolución digital, sustentada en la inteligencia artificial y en la robótica, ciertamente amenazaría puestos de trabajo ocupados por personas con niveles de cualificación media o elevada, y podría tener un efecto disruptivo. Es decir, esta vez podría ser distinto, porque evidentemente la evolución anterior del mercado laboral y la influencia que tuvieron en el pasado sus factores determinantes no deciden necesariamente su comportamiento futuro.

Es obvio, del mismo modo que ha sucedido siempre, que la nueva era de cambio tecnológico también inducirá ajustes importantes en el mercado laboral. Estas consecuencias serán heterogéneas, en función de la capacidad de los trabajadores para adaptarse a las tecnologías emergentes y de las tareas que estén desarrollando en su puesto de trabajo. De hecho, este es el elemento crítico en el análisis del impacto sobre el mercado laboral: ser conscientes de que las tecnologías emergentes no sustituyen empleos o puestos de trabajo, sino que lo que hacen es reemplazar tareas o conjuntos de tareas. Es decir, las tecnologías 4.0 afectan más a tareas específicas que no a cualificaciones concretas. Dos personas con la misma cualificación y el mismo tipo de empleo en dos empresas distintas, en la práctica probablemente están desarrollando tareas distintas en su puesto de trabajo, de manera que el impacto del cambio tecnológico probablemente será también desigual.

El foco de atención de las consecuencias del cambio tecnológico en el mercado laboral se debería orientar hacia las tareas que se desarrollan y hacia las habilidades que se requieren en cada puesto de trabajo, porque la adaptación al cambio tecnológico se llevará a cabo principalmente mediante la modificación en la estructura de las tareas en cada puesto de trabajo. El consenso académico existente nos muestra que el progreso tecnológico esencialmente presenta una tendencia a sustituir tareas rutinarias, mientras que la complementariedad de las habilidades requeridas estará definida por su naturaleza cognitiva o manual. La tabla siguiente nos ofrece, esquemáticamente, esta predicción. 
Tabla 1. Tipologías de tareas

\begin{tabular}{|c|c|c|c|}
\hline \multicolumn{4}{|c|}{ Complementariedad en las habilidades } \\
\hline \multirow{3}{*}{ Facilidad de automatización } & & Elevada & Baja \\
\hline & Elevada & Rutinarias cognitivas & Rutinarias manuales \\
\hline & Baja & No rutinarias cognitivas & No rutinarias manuales \\
\hline \multicolumn{4}{|c|}{ Impacto esperado del cambio tecnológico } \\
\hline \multicolumn{2}{|c|}{ Tipo de ocupación (según intensidad de habilidades) } & $\begin{array}{l}\text { Impacto esperado sobre la ocu- } \\
\text { pación }\end{array}$ & Impacto esperado sobre las rentas \\
\hline \multicolumn{2}{|l|}{ No rutinarias cognitivas } & Positivo & Positivo \\
\hline \multicolumn{2}{|c|}{ Rutinarias manuales y cognitivas } & Negativo & Negativo \\
\hline \multicolumn{2}{|l|}{ No rutinarias manuales } & Positivo & Negativo \\
\hline
\end{tabular}

Fuente: Elaboración propia a partir de WTO (2017)

De este modo, es previsible que la tecnología mejore las perspectivas de ocupación de las personas que realizan tareas no rutinarias y que implican habilidades cognitivas, ya que son menos fáciles de reproducir mediante algoritmos, a la vez que son más productivas con el apoyo de las tecnologías digitales. En cambio, el escenario es menos favorable para las tareas rutinarias que pueden sustituirse mediante la automatización digital, tanto si requieren de habilidades mentales como manuales. En cambio, el riesgo sería inferior para los puestos de trabajo definidos por un conjunto de tareas que, a pesar de ser manuales, no sean rutinarias, porque no son fácilmente automatizables a pesar de que puedan estar ocupados por trabajo poco cualificado.

Por lo tanto, se anunciaría una tendencia hacia la polarización creciente del mercado laboral, con una demanda relativa inferior de los niveles intermedios de cualificación, a la vez que se prevé un aumento en la desigualdad de las rentas salariales en favor del trabajo más cualificado. Diferentes motivos lo justificarían. Por un lado, como resultado de una mayor demanda relativa de los trabajadores con niveles educativos más altos. Por otro lado, por el desajuste existente en el mercado laboral entre oferta y demanda de habilidades. Estos desajustes (mismatch) entre las habilidades que demandan las empresas y los conocimientos disponibles por la fuerza laboral pueden dar lugar al desplazamiento del trabajo con más formación a lo largo de la escalera ocupacional, en detrimento de las personas con menor nivel educativo (deskilling).

\section{Evolución reciente del mercado laboral en España}

Una buena manera de aproximarse a cuáles serían los primeros efectos de esta nueva fase de progreso tecnológico sería analizar la evolución reciente del mercado laboral en España, porque se trata de una de las economías más afectadas por la reciente crisis financiera. El proceso de devaluación interna de costes tuvo en la destrucción de casi cuatro millones de puestos de trabajo una de sus principales consecuencias. La posterior reactivación económica ha renovado casi el $20 \%$ del mercado laboral en España, de manera que vale la pena analizar las características de la nueva ocupación generada, con el fin de inferir de qué transformaciones de la estructura ocupacional se acompaña la nueva etapa tecnológica. 
El estudio de los cambios en la distribución del trabajo, en función del nivel educativo y del tipo de ocupación, nos proporciona resultados relevantes. La evolución del mercado laboral en España, de acuerdo con los datos contenidos en la Encuesta de Población Activa, confirmaría que el cambio tecnológico tiene un efecto heterogéneo en la ocupación en función del nivel educativo. En general, podemos percibir que el mercado laboral español presenta algunos rasgos específicos que la reactivación posterior a la crisis financiera ha consolidado.

Un primer efecto muy visible es que la demanda de cualificaciones ha aumentado significativamente, de manera que parecería que el riesgo de automatización es más elevado entre las personas que realizan trabajos que requieren un nivel de cualificación laboral más bajo. Uno de cada dos nuevos puestos de trabajo creados es ocupado por una persona con estudios superiores. De este modo, el peso de la ocupación más cualificada en el mercado laboral en España supera el 43\% del empleo actual.

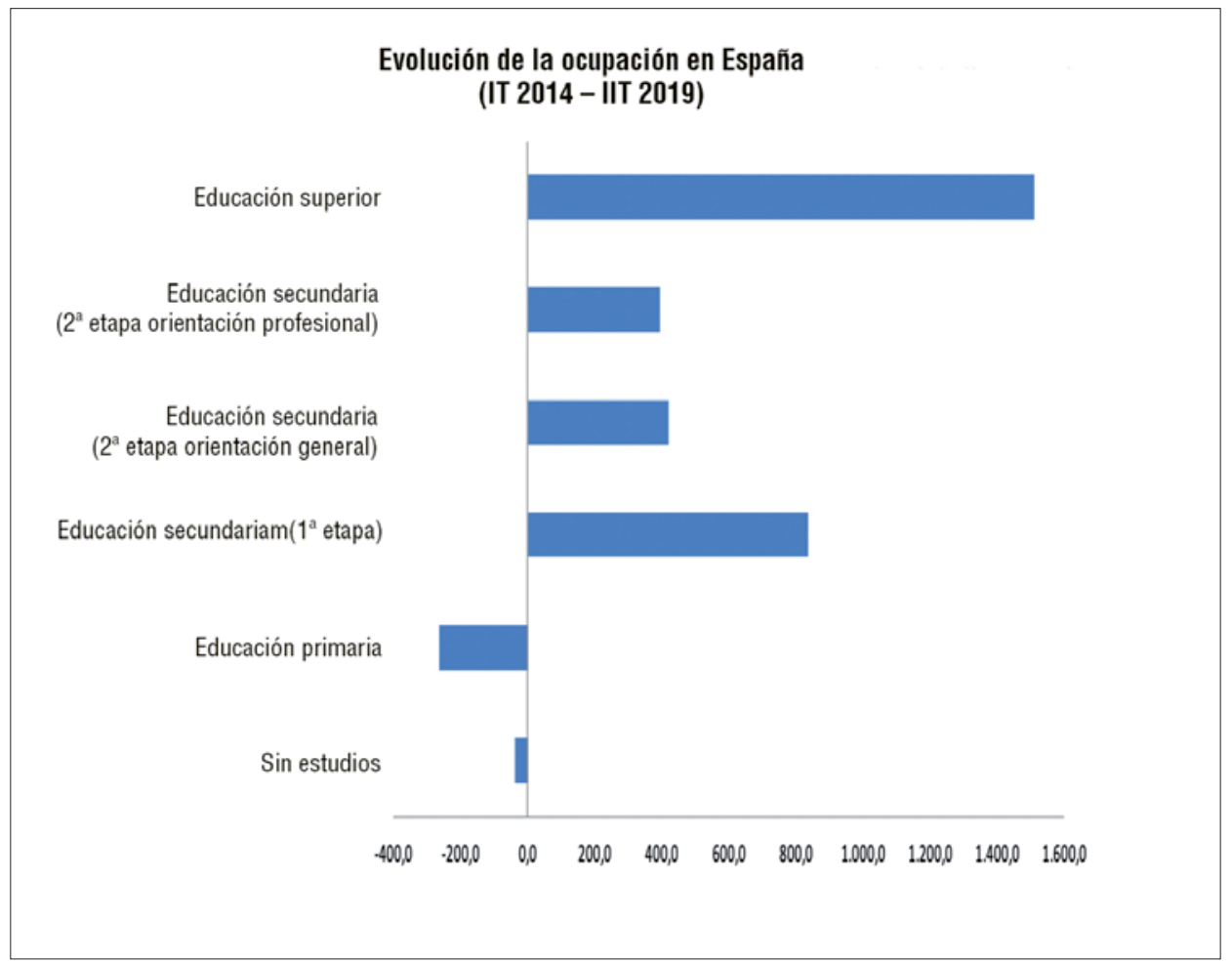

Fuente: Elaboración propia a partir de los datos de la Encuesta de Población Activa.

Pero más que un sesgo en favor del trabajo más cualificado, se puede percibir un creciente efecto de polarización, en la medida que a la vez que ha aumentado sustancialmente la ocupación más cualificada, el trabajo con niveles de cualificación inferiores también crece notablemente. En cambio, los niveles intermedios de cualificación estarían siendo los menos dinámicos en cuanto a la creación de nuevos puestos de trabajo, es decir, la nueva ocupación generada a partir de la reactivación económica habría consolidado las diferencias en la probabilidad de acceder a un trabajo en función del nivel educativo.

Una explicación de este comportamiento dual emerge cuando analizamos los perfiles profesionales demandados en la nueva ocupación. Se pone de manifiesto un crecimiento apreciable tanto de los puestos de trabajo que requieren un nivel elevado de cualificación profesional (técnicos y profesionales científicos e intelectuales...) como también de aquellos otros integrados mayoritariamente por tareas y funciones de carácter rutinario y, por lo tanto, potencialmente más susceptibles de verse afectados por el cambio tecnológico (trabajadores de servicios de restauración, ocupaciones elementales...). Este es un indicio del protagonismo, en la recuperación reciente, de la ocupación en algunas actividades económicas de bajo valor añadido. 


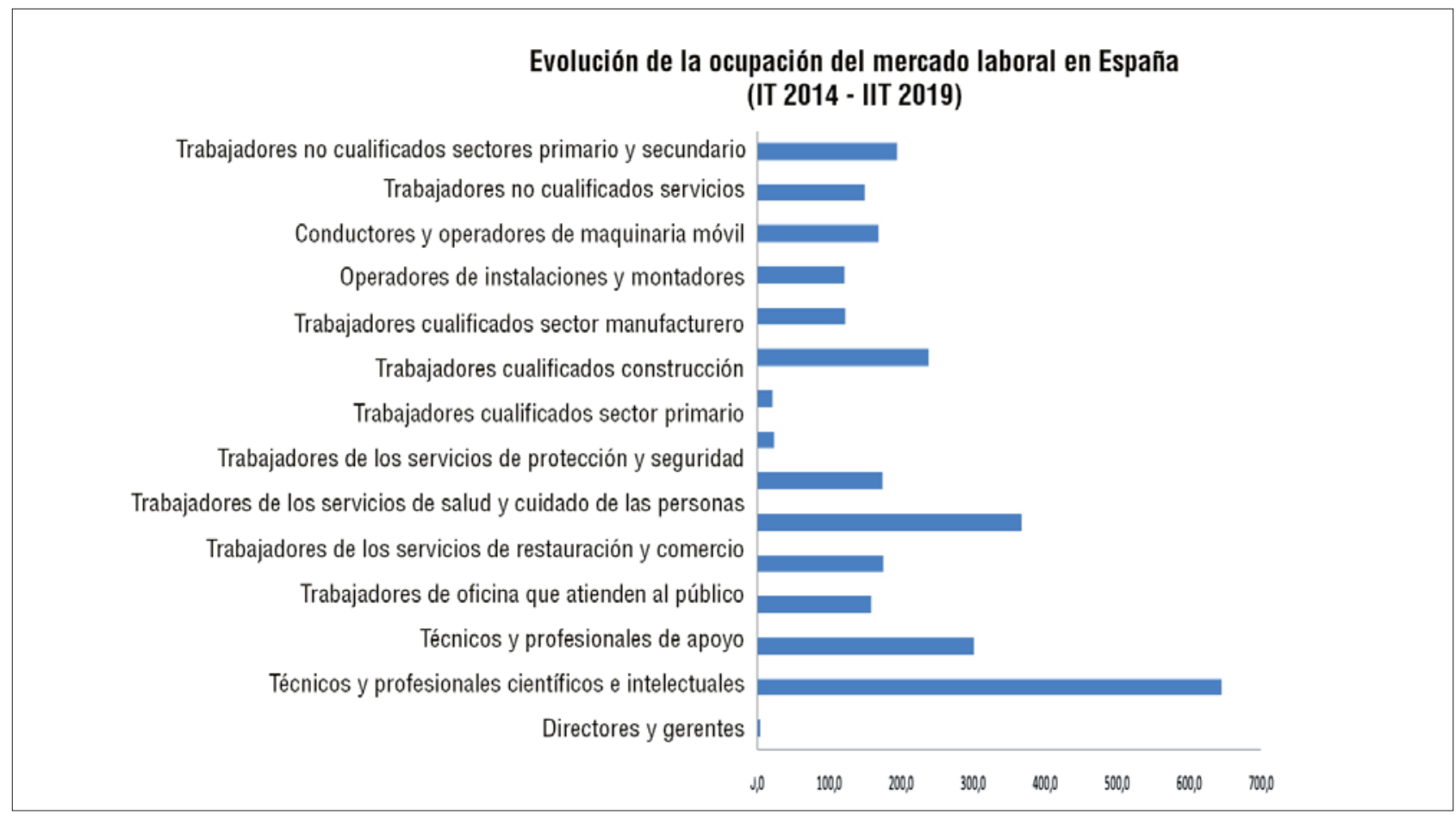

Fuente: Elaboración propia a partir de los datos de la Encuesta de Población Activa.

El análisis de los cambios recientes en el mercado laboral español se puede completar observando la demanda de habilidades requeridas en los nuevos puestos de trabajo creados. Inferimos esta demanda a partir de la nueva clasificación internacional de ocupaciones ISCO-08, que define cada trabajo a partir de las habilidades requeridas en los distintos tipos de ocupaciones para desarrollar eficazmente las tareas y las responsabilidades asignadas. Podemos observar cómo, después del ajuste y de la reactivación del mercado laboral, y a pesar de la magnitud de los nuevos puestos de trabajo creados, la estructura ocupacional no ha sufrido una transformación profunda en cuanto a las habilidades requeridas. Por el contrario, la demanda de habilidades básicas continúa predominando en la creación de nueva ocupación en España, lo que revela la resistencia al cambio del modelo productivo dominante. Es decir, el comportamiento reciente del mercado laboral pone de manifiesto que la creciente demanda de cualificaciones no se acompaña de una demanda mayoritaria de habilidades complejas. Es significativo que más del $40 \%$ de los puestos de trabajo de nueva creación son para ocupaciones que requieren habilidades básicas. La consecuencia inmediata de ello es un desajuste creciente vinculado a la demanda de cualificaciones. 


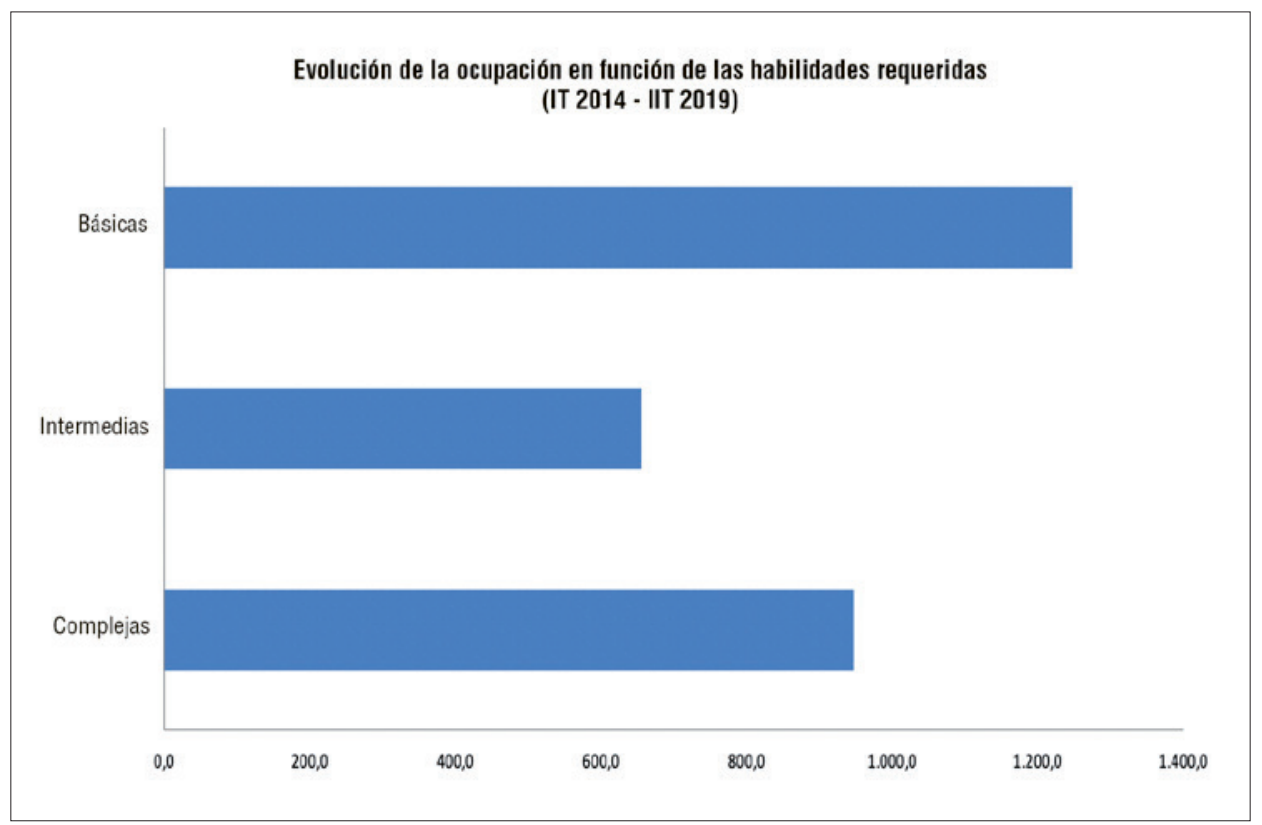

Fuente: Elaboración propia a partir de los datos de la Encuesta de Población Activa.

De este modo, a pesar de que la ocupación con estudios superiores es claramente predominante, la demanda relativa de habilidades complejas es mucho menos importante. El resultado inmediato es un desajuste creciente en función del nivel educativo con un desplazamiento progresivo de trabajadores con altos niveles de estudios hacia trabajos que requieren competencias y habilidades más sencillas, y que tradicionalmente ocupaban trabajadores menos cualificados. En consecuencia, a la vez que el mercado laboral se segmenta en favor de los más cualificados, el desencaje existente entre las cualificaciones de la demanda laboral y los requerimientos del sistema productivo induce a un desplazamiento descendente del trabajo más cualificado por la escalera ocupacional.

Otro efecto de que las oportunidades de trabajo sean más elevadas para el trabajo cualificado, pero que este ocupe de manera creciente puestos de trabajo de escaso valor añadido, sería la reducción de la prima salarial que reciben las personas con más estudios. Los datos de la Encuesta de estructura salarial nos muestran la tendencia existente en favor de una mayor remuneración de algunos perfiles menos demandados, pero que tienen restricciones de oferta por parte del sistema educativo, principalmente en cuanto a la formación profesional de grado superior. Este desajuste también incidiría en los cambios observados en la remuneración de las habilidades requeridas.

Al no disponer de datos posteriores a 2014, sería necesario un análisis más actualizado de la evolución salarial en función de los perfiles profesionales y de los niveles educativos para confirmar que, a medida que el trabajo más cualificado se desplaza por la escalera ocupacional, quedan afectadas tanto las oportunidades de encontrar trabajo como las rentas salariales. 


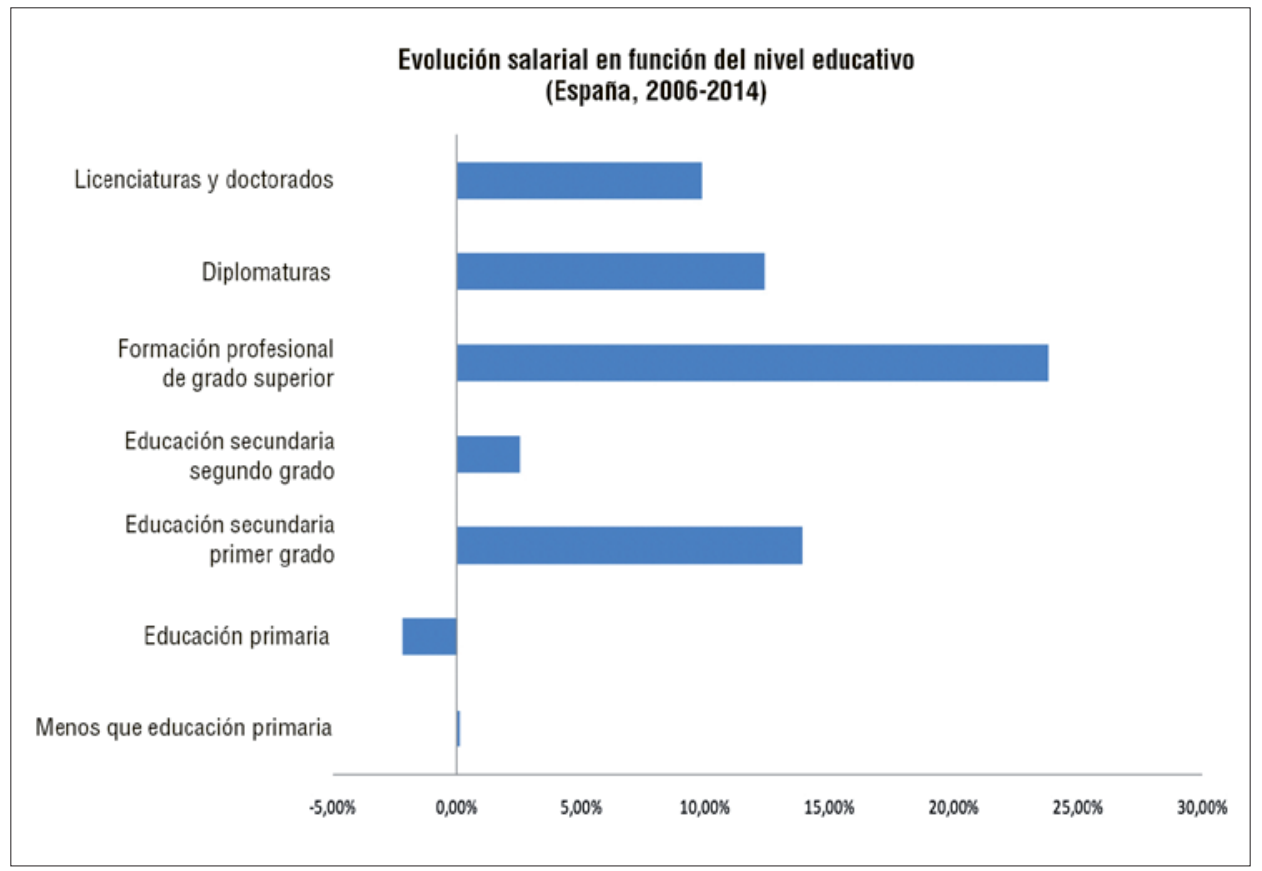

Fuente: Elaboración propia a partir de los datos de la Encuesta de estructura salarial.

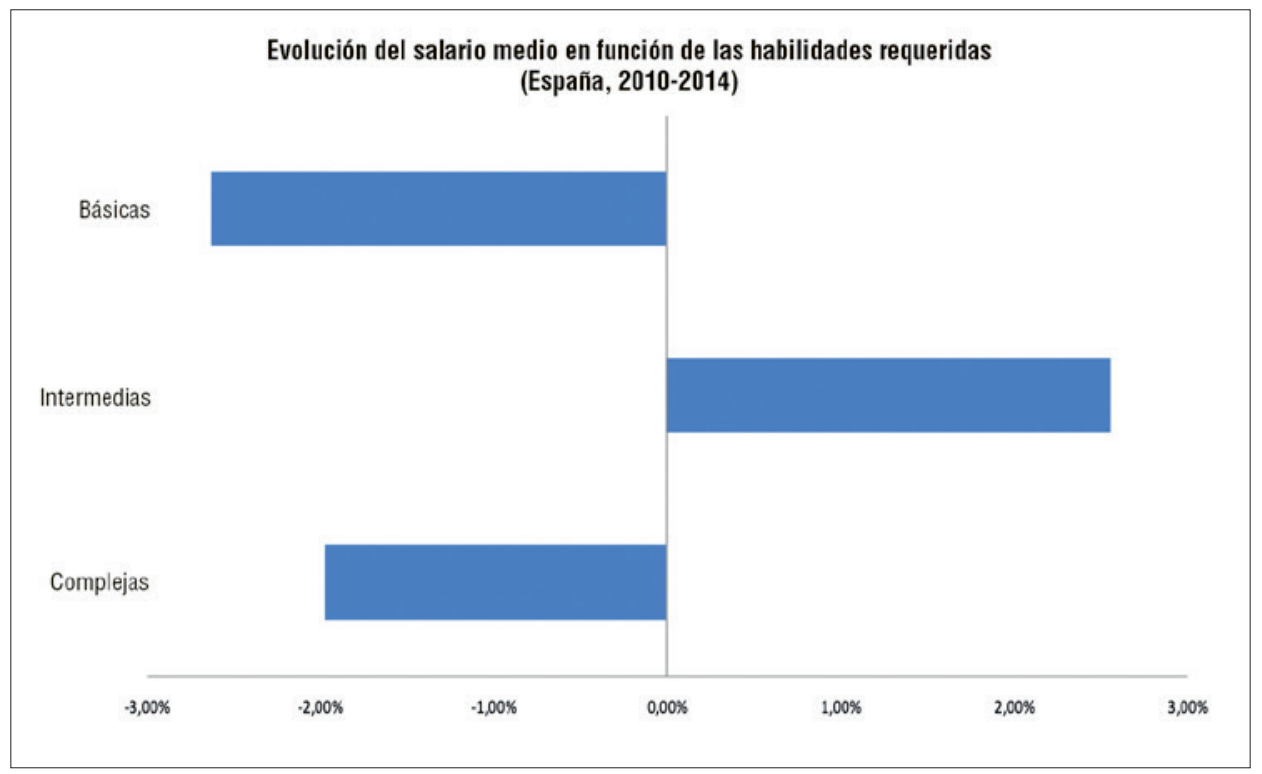

Fuente: Elaboración propia a partir de los datos de la Encuesta de estructura salarial.

En el contexto europeo, la economía española presenta una estructura económica que es relativamente más intensiva en puestos de trabajo que desarrollan tareas rutinarias. Pero a pesar de que en muchas economías europeas las tecnologías emergentes están instigando un cambio tecnológico que reduce la presencia de esta tipología de tareas, este no sería exactamente el caso del mercado laboral español. El principal motivo radica en el hecho de que en el modelo productivo predominen tareas que si bien son rutinarias no son altamente repetitivas, lo que complica su automatización. En general, son tareas llevadas a cabo sobre todo en los puestos de trabajo característicos de los servicios personales, como el turismo, la atención a las personas dependientes o la restauración. Esta mayor complejidad en la sustitución hombre-máquina limitaría el alcance de la automatización digital en España, a pesar de que la mayor parte de estos puestos de trabajo ocupen a personas con niveles educativos 
bajos. Hasta el momento, la transformación digital avanza lentamente en esta economía, probablemente también porque afronta la dura competencia de modelos de negocio basados en la contención salarial y en las precarias condiciones laborales de las personas que desarrollan este tipo de tareas. El conjunto de estos factores puede retrasar el impacto directo de la automatización digital en España, pero no evitar la polarización de las rentas salariales ni impedir el efecto sustitución en el futuro.

Probablemente la sociedad española está a las puertas de una transformación gradual y profunda de la economía y de su mercado laboral como resultado de la cuarta revolución industrial. En este momento, presenta algunos factores de riesgo asociados al cambio tecnológico. Es cierto que las oportunidades de ocupación han mejorado mucho para las personas más cualificadas, y que estas serán las más preparadas para desarrollar, programar y utilizar las nuevas tecnologías, pero también lo es que actualmente muchos trabajadores cualificados no pueden beneficiarse de su inversión en educación debido a la escasa demanda de sus habilidades en el mercado laboral español. Tampoco ayuda el menor uso relativo de las tareas cognitivas y de los recursos humanos expertos en ciencia y tecnología, los déficits organizativos y tecnológicos en el tejido productivo y un mismatch en el mercado laboral que evita que la experiencia laboral mejore sensiblemente el nivel competencial de los ocupados.

\section{Conclusiones}

Ciertamente, las innovaciones tecnológicas no aparecen nunca en el espacio vacío, sino en un entorno normativo y regulador concreto. Los nuevos desarrollos y aplicaciones exigirán cambios en el marco legal vigente y posiblemente inducirán a nuevas áreas normativas que acabarán definiendo la manera cómo las nuevas tecnologías podrán utilizarse. Del mismo modo, la respuesta social a un cambio que es potencialmente disruptivo no solo será lo que definirá los usos finales y delimitará el alcance de las nuevas tecnologías, sino que también influirá en la orientación y en los contenidos reguladores. La viabilidad técnica y económica no es suficiente si no se promueve la adopción de nuevas regulaciones que generen un mayor clima de confianza, seguridad y aceptación social.

No todo lo que percibimos como tecnológicamente posible será económicamente viable, rentable o aceptable para las empresas, que tendrán que afrontar las restricciones internas de los recursos y gestionar sus procesos de toma de decisiones estratégicas en el contexto de su propia cultura directiva y organizativa. La velocidad de adopción de las nuevas tecnologías a menudo es más incierta y volátil de lo que a priori aparenta.

No hace falta, pues, precipitarse a la hora de realizar valoraciones sobre los efectos futuros en el mercado laboral, pero la composición del empleo muestra una tendencia a la segmentación, y el sesgo sobre las oportunidades de trabajo ya es evidente, de manera que el riesgo asociado a la automatización digital se irá distribuyendo desigualmente entre la sociedad. En estos casos, son más trascendentes y necesarias las políticas que ayudan a preparar a las personas para los cambios en los requisitos laborales. Parecería adecuado afinar en las prioridades y en los contenidos de las políticas públicas y en las estrategias empresariales para facilitar una atención adecuada a un reto que es complejo y saber sacar el máximo provecho social a la vez mediante una apuesta colectiva que fuera socialmente más inclusiva.

Desde la perspectiva de las políticas públicas, probablemente sería conveniente focalizar los esfuerzos en facilitar la actividad de investigación y de desarrollo en tecnologías relacionadas con la inteligencia artificial, promover mecanismos de impulso al cambio tecnológico, favorecer la diseminación de las tecnologías emergentes en condiciones de libre concurrencia, educar a la sociedad para el trabajo futuro, favorecer la formación en ocupaciones emergentes, mejorar el capital humano facilitando la formación en el puesto de trabajo, promover la flexibilidad interna en las organizaciones, reforzar la red de protección social y la transición entre ocupaciones laborales o impulsar políticas de inversión que garanticen el acceso universal a las infraestructuras digitales, entre otros.

En cuanto a las estrategias empresariales, tal vez parecerían necesidades urgentes mejorar la dotación interna de capital humano y tecnológico, promover cambios organizativos que consoliden una ocupación de más calidad, modificar los contenidos de las políticas de formación interna, estimular la cooperación orientada al desarrollo y a la aplicación de innovaciones, acelerar la creación de puestos de trabajo digitales, experimentar con puestos de trabajo basados en la interacción seres humanos-máquinas, promover la adaptación al cambio 
tecnológico como elemento de diferenciación en el mercado y desarrollar soluciones tecnológicas que permitan atender necesidades específicas de los consumidores.

Si la innovación tecnológica es fuente de progreso económico y motor de cambio social, y si la ciencia y los conocimientos de hoy se convertirán en la tecnología del mañana, las líneas de juego están bien diseñadas para que las instituciones públicas y el tejido empresarial interactúen con habilidad para vencer los obstáculos, los costes y las dificultades inherentes al cambio, y transformar el desafío 4.0 en un triunfo para el conjunto de la sociedad.

\section{Algunas referencias para saber más cosas...}

ACEMOGLU, D.; AUTOR, D. (2011). «Skills, Tasks and Technologies: Implications for Employment and Earnings». En: David Cardo; Orley Ashenfelter (eds). Handbook of Labor Economics. Vol. 4b, págs. 1043-1171. https:// doi.org/10.1016/S0169-7218(11)02410-5

ACEMOGLU, D.; RESTREPO, P. (2017). «Robots and Jobs: Evidence from US Labor Markets». NBER Working Papers. National Bureau of Economic Research. MIT. https://doi.org/10.3386/w23285

ARNTZ, M; GREGORY, T.; ZIERAHN, U. (2016). «The Risk of Automatization for Jobs in OECD Countries: A Comparative Analysis». OECD Social, Employment and Migration Working Papers. N.o 189. París. https://doi. org/10.1787/5jlz9h56dvq7-en

AUTOR, D. H. (2015). «Why Are There Still So Many Jobs? The History and Future of Workplace Automation». Journal of Economic Perspectives. Vol. 29, n.o 3, págs. 3-30. https://doi.org/10.1257/jep.29.3.3

AUTOR, D. H.; LEVY, F.; MURNAME, R. J. (2003). «The Skill Content of Recent Technological Change». The Quarterly Journal of Economics. Vol. 118, n.o 4, págs. 1279-1333. https://doi.org/10.1162/003355303322552801

BLANCO, R.; FONTRODONA, J.; POVEDA, C. (2016). «La indústria 4.0 a Catalunya». Memòria Econòmica de Catalunya, 2016. Consell de Cambres de Comerç de Catalunya.

BOSTON CONSULTING GROUP (2015a). Industry 4.0: The Future of Productivity and Growth in Manufacturing Industries.

BOSTON CONSULTING GROUP (2015b). Man and Machine in Industry 4.0. How Will Technology Transform the Industrial Workforce Through 2025 ?

CAIXABANK RESEARCH (2016). «Les noves tecnologies i el mercat de treball». [dosier incluido en el Informe Mensual, 398].

DELOITTE (2015). From brawn to brains. The impact of technology on jobs in the UK.

EUROFOND (2016). What do Europeans do at work? A task-based analysis: European Jobs Monitor 2016. Eurofound European Foundation for the Improvement of Living and Working Conditions.

FREY, C. B.; OSBORNE, M. (2013). «The Future of Employment: How Susceptible are Jobs to Computerization?». Working Paper. Oxford Martin School: University of Oxford.

GRAETZ, G.; MICHAELS, G. (2015). «Robots at Work». CEP Discussion Paper. N.o 1335.

GREGORY, T.; SALONOMS, A.; ZIERAHN, U. (2016). «Racing With or Against the Machine? Evidence from Europe». Discussion Paper. N.o 16-053. Centre for European Economic Research. https://doi.org/10.2139/ ssrn.2815469

MARCOLIN, L.; MIROUDOT, S.; SQUICCIARINI, M. (2016). «Routine Jobs, employment and technological innovation in global value chains». OECD Science, Technology and Industry Working Papers. OECD Publishing. https://doi.org/10.1787/5jm5dcz2d26j-en

MCKINSEY GLOBAL INSTITUTE (2017). «Artificial Intelligence: The Next Digital Frontier?». Discussion Paper. https://doi.org/10.1016/S1353-4858(17)30039-9

MOKYR, J.; VICKERS, C.; ZIEBARTH, N. L. (2015). «The History of Technological Anxiety and the Future of Economic Growth: Is This Time Different?». Journal of Economic Perspectives. Vol. 29, n.o 3, págs. 31-50. https:// doi.org/10.1257/jep.29.3.31 
RANDSTAD RESEARCH (2016). La digitalización: ¿crea o destruye empleo? Informe anual sobre la flexibilidad laboral y el empleo.

UNCTAD (2017). «Robots, Industrialization and Inclusive Growth». Trade and Development Report 2017. Beyond Austerity: Towards a Global New Deal. Págs. 35-66.

WORLD TRADE ORGANIZATION (2017). World Trade Report 2017: Trade, technology and jobs.

Cita recomendada: LLADÓS, Josep. ¿Nos robarán los robots los puestos de trabajo? Un vistazo al mercado laboral en España. Oikonomics [en línea]. Noviembre 2019, no. 12, pp. 1-11. ISSN: 2339-9546. DOI: https://doi.org/10.7238/o.n12.1911

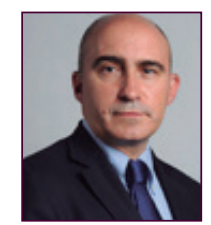

\section{Josep Lladós}

jlladosm@uoc.edu

\section{Profesor agregado de los Estudios de Economía y Empresa de la UOC}

Josep Lladós es licenciado y doctor en Ciencias Económicas y Empresariales, profesor agregado de los Estudios de Economía y Empresa de la UOC, director del programa interuniversitario del Doctorado en Administración y Dirección de Empresas, e investigador del grupo de investigación DigiBiz (http://transfer.rdi.uoc.edu/es/grupo/digital-business-research-group). Su área de conocimiento es la economía aplicada, y focaliza su actividad de investigación principalmente en los ámbitos de la economía internacional, la geografía económica y los procesos de innovación empresarial.

Los textos publicados en esta revista están sujetos -si no se indica lo contrario- a una licencia de Reconocimiento 4.0 Internacional de Creative Commons. Puede copiarlos, distribuirlos, comunicarlos públicamente, hacer obras derivadas siempre que reconozca los créditos de las obras (autoría, nombre de la revista, institución editora) de la manera especificada por los autores o por la revista. La licencia completa se puede consultar en https://creativecommons.org/licenses/by/4.0/deed.es_ES.

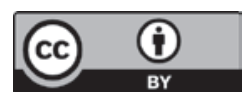

